\title{
Expression of methylation-modulated tumor-related genes in endoscopically resected early esophageal squamous neoplasia
}

\author{
KOHEI HOSODA, KAZUO YASHIMA, AKIHIRO TAMOTO, SOHEI YAMAMOTO, \\ SOICHIRO KAWATA, YUICHIRO IKEBUCHI, KAZUYA MATSUMOTO, KOICHIRO KAWAGUCHI, \\ KENICHI HARADA, YOSHIKAZU MURAWAKI and HAJIME ISOMOTO
}

Division of Medicine and Clinical Science, Faculty of Medicine, Tottori University, Yonago, Tottori 683-8504, Japan

Received August 14, 2016; Accepted March 28, 2017

DOI: $10.3892 / \mathrm{ol} .2017 .6196$

\begin{abstract}
Smoking and alcohol consumption are major risk factors for the development of esophageal squamous cell carcinoma (ESCC). Recent studies have demonstrated that smoking and alcohol consumption may be associated with altered DNA methylation in human cancer development. The aim of the present study was to evaluate methylation-modulated protein expression of tumor-related genes (TRGs) in the early stages of esophageal squamous neoplasia (ESN). ESN tissue samples $(n=141)$ comprising 19 cases of low-grade intraepithelial neoplasia (LGIN), 70 of high-grade intraepithelial neoplasia/carcinoma in situ (HGIN/CIS) and 52 of invasive cancer, were endoscopically resected. The methylation-modulated protein expression of 5 TRGs [fragile histidine triad (FHIT), E-cadherin, MutL homolog 1 (MLH1) /MutS homolog 2 (MSH2) and cyclooxygenase-2 (COX-2)] as well as p53 was examined with immunohistochemistry, and their expression was compared with patient clinicopathological characteristics. Reduced or loss of FHIT, E-cadherin, MLH1/MSH2 and COX-2 expression was detected in 26.3 (5/19), $5.3(1 / 19), 0(0 / 19)$ and $63.2 \%(12 / 19)$ of LGIN cases, 61.4 (43/70), 18.6 (13/70), 7.1 $(5 / 70)$ and $65.7 \%(46 / 70)$ of HGIN/CIS cases, and $78.8(41 / 52)$, $50.0(26 / 52), 11.5(6 / 52)$ and $59.6 \%(31 / 52)$ of invasive cancer cases, respectively. Reduced or absent expression of FHIT and E-cadherin was significantly associated with neoplastic progression (FHIT, $\mathrm{P}=0.0007$; E-cadherin, $\mathrm{P}=0.00014$ ). The mean number of TRGs (FHIT, E-cadherin, MLH1/MSH2, and $C O X-2)$ that exhibited reduced or absent expression in LGIN, HGIN/CIS and invasive cancer specimens was $1.12 \pm 0.61$, $1.66 \pm 0.93$ and $2.09 \pm 0.96$, respectively, demonstrating a significant stepwise increment from LGIN to HGIN/CIS and then to invasive cancer $(\mathrm{P}<0.05)$. p53 overexpression was
\end{abstract}

Correspondence to: Dr Kazuo Yashima, Division of Medicine and Clinical Science, Faculty of Medicine, Tottori University, 36-1 Nishicho, Yonago, Tottori 683-8504, Japan

E-mail: yashima@med.tottori-u.ac.jp

Key words: esophageal cancer, endoscopic resection, fragile histidine triad, E-cadherin, MutL homolog 1 frequently detected in ESN with head and neck carcinomas. However p53 overexpression was not significantly associated with ESN progression. An increase in the number of the 5 TRG proteins with reduced or loss of expression in the early stages of esophageal tumorigenesis was demonstrated, and their decreased expression was observed to be associated with tumor progression. Therefore, smoking and alcohol drinking may be associated with not only carcinogenesis but also the progression of ESN.

\section{Introduction}

In Japan, esophageal squamous cell carcinoma (ESCC) accounts for $>90 \%$ of esophageal cancer cases (1). As ESCC is frequently detected at an advanced stage, it has one of the worst prognoses among digestive carcinomas (1). Therefore, early detection of malignant changes in the esophageal epithelium is the most effective way to improve the prognosis for ESCC. Detection at an early stage is gradually becoming more feasible due to advances in endoscopy, and the number of cases that can be potentially treated endoscopically is increasing (2).

A variety of genetic and epigenetic alterations are associated with esophageal carcinoma $(3,4)$, including mutation of the p53 gene $(5,6)$. A number of previous studies have concluded that $p 53$ is the most frequently mutated gene in $\operatorname{ESCC}(7,8)$. The role of epigenetic changes, including aberrant DNA methylation, is particularly significant in human cancer development $(9,10)$. An increasing number of tumor-related genes (TRGs) that are inactivated by the hypermethylation of $\mathrm{CpG}$ islands have been reported in esophageal cancer, including ESCC $(11,12)$. However, the role of hypermethylation of $\mathrm{CpG}$ islands in early neoplastic lesions is not well understood.

In esophageal carcinomas, FHIT, E-cadherin and $M L H 1 / M S H 2$ have been previously demonstrated to be inactivated through hypermethylation of their promoters (13-17). These genes may be inactivated by a combination of genetic or epigenetic alterations of two alleles. However, epigenetic change is likely to be the predominant mechanism associated with the loss of FHIT, E-cadherin and MLH1/MSH2 function in sporadic esophageal carcinomas (13-17).

Numerous epidemiological studies have identified tobacco smoking and alcohol consumption as the major risk factors for the development of ESCC $(18,19)$. Increasing evidence 
indicates that smoking and alcohol induce epigenetic alterations, particularly aberrant patterns of DNA methylation. Such alterations to methylation are induced by tobacco and alcohol consumption in the development of ESCC $(20,21)$, and may be important contributing factors in carcinogenesis $(20,22)$.

In the present study, the clinical significance of TRG protein expression was evaluated during the early stages of esophageal carcinogenesis. Previous studies have demonstrated that the DNA methylation status of the TRGs FHIT, E-cadherin, $M L H 1 / M S H 2$ and $C O X-2$ can be detected by immunohistochemical analysis of tumor specimens $(13-17,23)$. Therefore, the protein expression of these 5 TRGs and p53 was analyzed by immunostaining in 141 early esophageal neoplasia tumor specimens, comprising 19 low-grade intraepithelial neoplasia (LGIN), 70 high-grade intraepithelial neoplasia/carcinoma in situ (HGIN/CIS) and 52 invasive cancer tissue samples extracted during endoscopic resection.

\section{Patients and methods}

Patient and tissue samples. A total of 141 esophageal squamous neoplasia (ESN) tumor specimens were obtained from 112 patients (including 102 males and 10 females) who underwent endoscopic resection at Tottori University Hospital (Yonago, Japan) between January 2004 and December 2014. The age of the patients ranged from 43 to 85 years (mean, 68.6 years). None of the patients had received preoperative radiotherapy or chemotherapy. Based on a histological examination, the 141 tumor specimens were classified as 19 cases of low-grade dysplasia, 70 of high-grade dysplasia and 52 of invasive cancer confined to the lamina propria mucosa or submucosa based on the World Health Organization classification (24) (Table I). Among the 112 patients with ESN, 98 (87.5\%) had a history of smoking and $99(88.4 \%)$ had a history of drinking. A total of 5 patients (4.4\%) were neither smokers nor drinkers. All cases were analyzed anonymously, i.e., all specimens were assigned numbers without any associated personal information. Approval was obtained for the present study from the Institutional Ethics Review Board of Tottori University (grant nos. 314 and 1508A024; Yonago, Japan), which certified that the study complied with the Declaration of Helsinki and written informed consent was obtained from all patients.

Immunohistochemical staining. For immunohistochemical staining, paraffin-embedded, $4-\mu \mathrm{m}$-thick tumor sections were first deparaffinized in xylene and rehydrated in ethanol. Subsequently, the sections were immersed in citrate buffer $(0.01 \mathrm{M}$, $\mathrm{pH}$ 6.0) and heated in a microwave oven for 20-30 min for antigen retrieval. Endogenous peroxidase activity was blocked by incubation with $3 \% \mathrm{H}_{2} \mathrm{O}_{2}$ for $30 \mathrm{~min}$ at room temperature. The sections were subsequently incubated with a primary antibody overnight at $4^{\circ} \mathrm{C}$. The primary antibodies used included rabbit polyclonal anti-FHIT (dilution, 1:100; clone F130; cat. no. 18163; Immuno-Biological Laboratories Co. Ltd., Gunma, Japan), mouse monoclonal anti-p53 (dilution, 1:50; clone DO-7; cat. no. M7001; Dako; Agilent Technologies, Inc., Santa Clara, CA, USA), anti-E-cadherin (dilution, 1:50; clone HECD-1; catalog no. M106; Takara, Bio Inc., Otsu, Japan), anti-MSH2 (dilution, 1:100; clone FE11; cat. no. 33-7900; Oncogene
Table I. Clinicopathological characteristics and patient background of patients with early esophageal neoplasia.

\begin{tabular}{lc} 
Characteristic & $\mathrm{n}$ \\
\hline Gender & \\
Male & 102 \\
Female & 10 \\
Age, mean \pm SD, years & $68.6 \pm 8.7$ \\
Tumor size, $\mathrm{mm}$ & \\
$<10$ & 16 \\
$\geq 10$ to $<20$ & 51 \\
$\geq 20$ & 74
\end{tabular}

Tumor location

Upper 62

Middle 64

Lower $\quad 15$

Macroscopic tumor type

Elevated 18

Flat $\quad 59$

Depressed 64

Invasion depth

Low-grade intraepithelial neoplasia $\quad 19$

High-grade intraepithelial

neoplasia/carcinoma in situ $\quad 70$

Lamina propria invasion $\quad 27$

Muscularis mucosa invasion $\quad 11$

Submucosal invasion $\quad 14$

Alcohol history

Drinker $\quad 99$

Non-drinker 13

Smoking history

Current/previous smoker $\quad 98$

Never smoked 14

Brinkman index ${ }^{\mathrm{a}}$, mean \pm SD $\quad 791 \pm 689$

History of head and neck cancer 9

A total of 141 samples were used from 112 patients. SD, standard deviation. ${ }^{\mathrm{a} B r i n k m a n}$ index: number of cigarettes/day x years.

Research Products; EMD Millipore, Billerica, MA, USA), anti-MLH1 (dilution, 1:50; clone G168-15; cat. no. 550838; BD Pharmingen, San Diego, CA, USA) and anti-COX-2 (dilution, 1:100; clone 33/Cox-2; cat. no. 610204; BD Biosciences, Franklin Lakes, NJ, USA) antibodies. As a negative control, the primary antibody was replaced with normal rabbit IgG (cat. no. GTX35035; Gene Tex, Irvine, CA, USA) or normal mouse IgG (cat. no. NC494H; Biocare Medical, Pike Lane Concord, CA, USA) at a similar dilution. Antibody binding was detected by incubating with anti-rabbit (dilution, 1:200; cat. no. BA-1000; Vector Laboratories, Inc., Burlingame, CA, USA) or anti-mouse (dilution, 1:200; cat. no. BA-2000; Vector Laboratories, Inc., Burlingame, CA, USA) with avidin-biotin-peroxidase for $30 \mathrm{~min}$ at room temperature, and was then visualized using the chromogen diaminobenzidine 
A

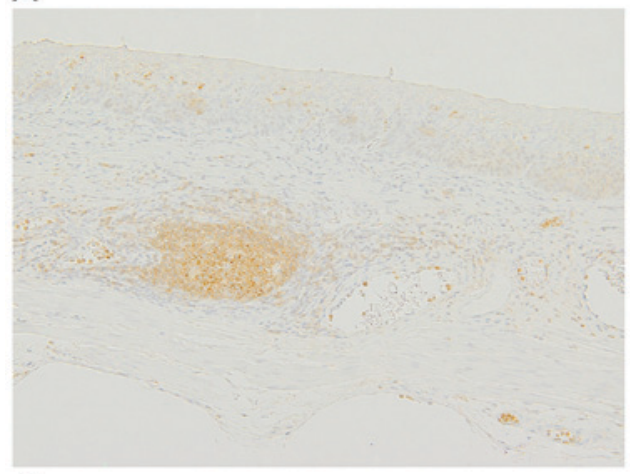

C

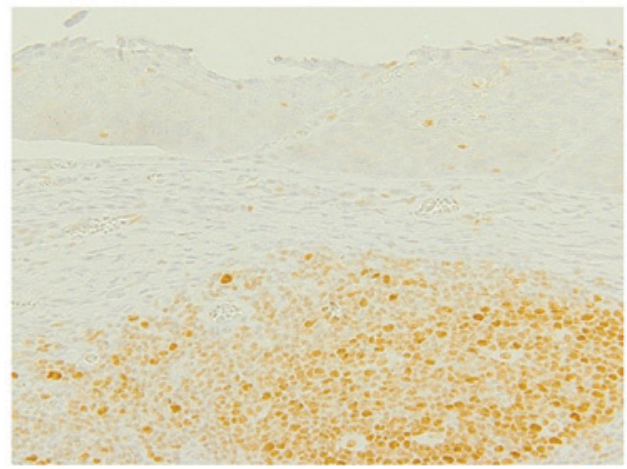

B

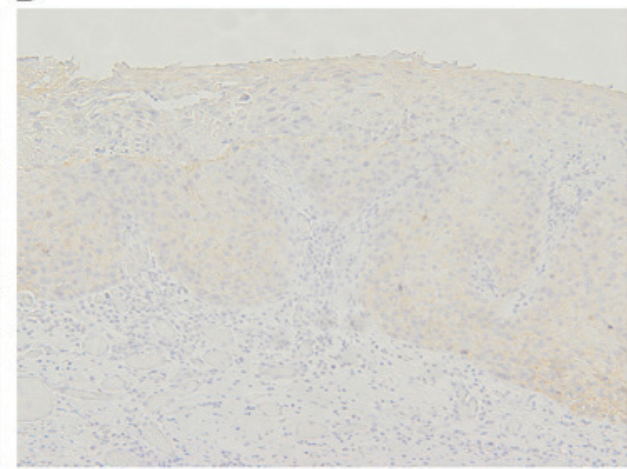

D

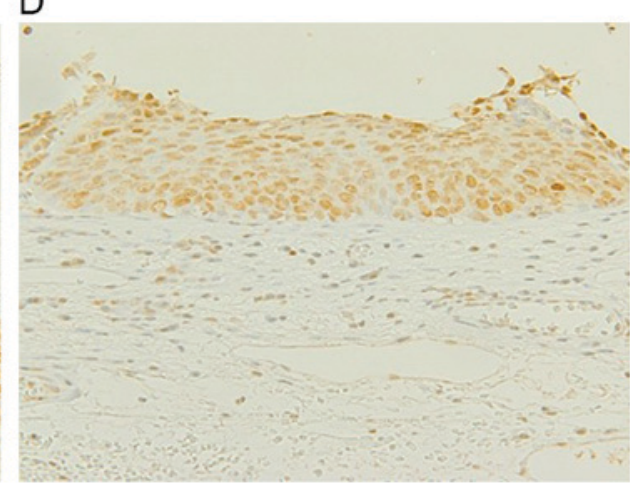

Figure 1. Representative immunostaining results of FHIT, E-cadherin, MLH1 and MSH2 in neoplastic tissue. Esophageal squamous neoplastic tissue obtained from endoscopic resection was stained with antibodies against FHIT, E-cadherin and MLH1/MSH2. (A) A HGIN/CIS lesion with reduced FHIT immunostaining, with positive staining of the adjacent germinal center and infiltrating lymphocytes. (B) A HGIN/CIS lesion with negative E-cadherin immunostaining. (C) A HGIN/CIS lesion with negative MLH1 immunostaining. (D) A HGIN/CIS lesion with positive MSH1 immunostaining. FHIT, fragile histidine triad; MLH1, MutL homolog 1; MSH1, MutS homolog 1; HGIN/CIS, high-grade intraepithelial neoplasia/carcinoma in situ .

tetrahydrochloride (cat. no. SK-4100; Vector Laboratories, Inc., Burlingame, CA, USA). The signal was detected as described in the Vectastain Elite avidin-biotin complex kit protocol (Vector Laboratories, Inc., Burlingame, CA, USA). Hematoxylin was used as a counterstain.

For each specimen, $>5$ fields were viewed under a light microscope (magnification, x 100) (Olympus Corporation, Tokyo, Japan). Protein expression was evaluated by two independent observers. p53 overexpression was defined as a distinct nuclear immunoreaction in $>30 \%$ of the cells of the sample.

FHIT expression was graded, based on the intensity of cytoplasmic staining, as 'reduced', 'absent' or 'positive' as described previously (25). FHIT expression in normal esophageal squamous epithelia and normal esophageal glands served as positive controls. The adjacent normal squamous epithelium was used as an internal positive control. FHIT weak positive lesions showed positive reactivity at a level that was weak compared with the level in the normal epithelia, and these lesions were described as reduced.

E-cadherin, MLH1/MSH2 and COX-2 expression were classified as 'positive' or 'decreased'. Cases with staining (E-cadherin, membrane; MLH1/MSH2, nucleus; COX-2, cytoplasm) in $<30 \%$ of the tumor cells or with a complete absence of staining were categorized as decreased, respectively.

Statistical analysis. Statistical analysis was performed using the $\chi^{2}$ test with the Yates correction or the Newman-Keuls test. $\mathrm{P}<0.05$ was considered to indicate a statistically significant difference. All statistics were calculated using Stat Flex version 6.0 (Artech Co., Ltd, Osaka, Japan).

\section{Results}

Immunohistochemical analysis of the expression of TRGs (FHIT, E-cadherin, MLH1/MSH2 and COX-2) in ESN tumors. Representative images of immunohistochemical staining of FHIT, E-cadherin and MLH1/MSH2 in ESN tumors is included in Fig. 1, and the results are quantified in Table II. There was a reduced or loss of FHIT, E-cadherin, MLH1/MSH2 and COX-2 expression in $26.3 \%(5 / 19), 5.3 \%(1 / 19), 0 \%(0 / 19)$ and $63.2 \%(12 / 19)$ of LGIN cases, in $61.4 \%(43 / 70), 18.6 \%$ (13/70), 7.1\% (5/70) and 65.7\% (46/70) of HGIN/CIS cases, and in $78.8 \%$ (41/52), 50.0\% (26/52), 11.5\% (6/52) and $59.6 \%$ (31/52) of invasive cancer cases, respectively. The incidence of absent or reduced FHIT or E-cadherin expression was significantly associated with neoplastic progression from LGIN to HGIN/CIS to invasive cancer (FHIT, $\mathrm{P}=0.0007$; E-cadherin, $\mathrm{P}=0.00014)$. In the present study, absent or reduced protein expression of any of the 5 TRGs (FHIT, E-cadherin, MLH1, $M S H 2$ and $C O X-2)$ in early ESN samples was not associated with the expression of the other TRGs, or with other clinical parameters, including size, age, gender and tumor location (data not shown).

There was an increase in the mean number of the TRG proteins with reduced or loss of expression with increasing 
Table II. Analysis of FHIT, CDH1, MLH1/MSH2 and COX-2 protein expression in patients with early esophageal squamous neoplasia.

\begin{tabular}{lcccr}
\hline $\begin{array}{l}\text { Reduced or loss } \\
\text { of expression }\end{array}$ & LGIN, n (\%) & HGIN/CIS & Invasive cancer & Total \\
\hline Total samples, $\mathrm{n}$ & 19 & 70 & 52 & 141 \\
FHIT & $5(26.3)^{\mathrm{a}}$ & $43(61.4)^{\mathrm{a}}$ & $41(78.8)^{\mathrm{a}}$ & $89(63.1)$ \\
CDH1 & $1(5.3)^{\mathrm{b}}$ & $13(18.6)^{\mathrm{b}}$ & $26(50.0)^{\mathrm{b}}$ & $40(28.4)$ \\
MLH1 & $0(0)$ & $5(7.1)$ & $5(9.6)$ & $10(7.1)$ \\
MSH2 & $0(0)$ & $0(0)$ & $2(3.8)$ & $2(1.4)$ \\
COX-2 & $12(63.2)$ & $46(65.7)$ & $31(59.6)$ & $89(63.1)$
\end{tabular}

${ }^{\mathrm{a}} \mathrm{P}=0.0007,{ }^{\mathrm{b}} \mathrm{P}=0.0001 ; \chi^{2}$ test with Yates' correction. A total of 1 sample with reduced and loss of MLH1 and MSH2 expression. CDH1, E-cadherin; COX-2, cyclooxygenase-2; FHIT, fragile histidine triad; MLH1, MutL homolog 1; MSH2, MutS homolog 2; LGIN, low-grade intraepithelial neoplasia; HGIN/CIS, high-grade intraepithelial neoplasia/carcinoma in situ.

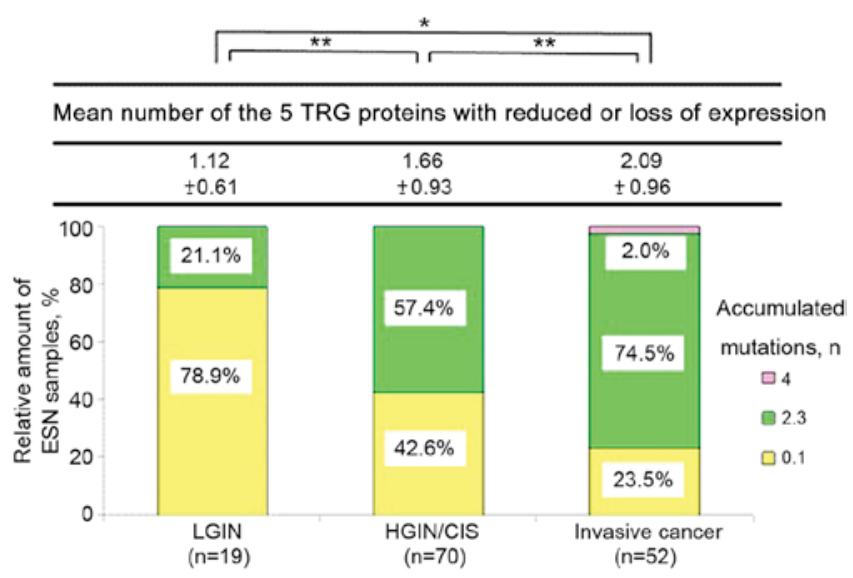

Figure 2. Number of TRGs (FHIT, CDH1, MLH1/MSH2 and COX-2) with negative expression in early esophageal squamous neoplasia. The number of TRG proteins for which the expression was decreased or absent in tumors gradually increased in the progression from LGIN and HGIN/CIS to invasive cancer. ${ }^{*} \mathrm{P}<0.01,{ }^{* *} \mathrm{P}<0.05$; Newman-Keuls test. Data are presented as the mean \pm standard deviation. $\mathrm{CDH} 1, \mathrm{E}$-cadherin; COX-2, cyclooxygenase-2; TRG, tumor-related genes; FHIT, fragile histidine triad; MLH1, MutL homolog 1; MSH1, MutS homolog 1; LGIN, low-grade intraepithelial neoplasia; HGIN/CIS, high-grade intraepithelial neoplasia/carcinoma in situ.

tumor progression from LGIN (mean \pm standard deviation, $1.12 \pm 0.61)$ to HGIN/CIS $(1.66 \pm 0.93)$ to invasive cancer (2.09 \pm 0.96 ; Fig. 2). The presence of a trend was evaluated using the Newman-Keuls test on the three groups (LGIN, HGIN/CIS and invasive cancer), which identified a significant increase in the number of the TRG proteins with reduced or loss of expression from LGIN to invasive cancer $(\mathrm{P}<0.05)$.

Immunohistochemical analysis of p53 in ESN tumors. p53 overexpression was detected in $42.1 \%$ (8/19) of LGIN cases, in $57.1 \%$ (40/70) of HGIN/CIS cases, and in $55.8 \%$ (29/52) of invasive cancer cases (data not shown). p53 overexpression was not associated with ESN progression. In addition, p53 overexpression was not associated with absent or reduced protein expression of any of the 5 TRGs or with the number of TRGs expressed. The percentage of tumors with p53 overexpression was higher in ESN with head and neck carcinoma
(HNC; 8/9; 88.9\%) compared with in ESN with other cancer $(25 / 50 ; 50.0 \%$; $\mathrm{P}=0.064)$.

\section{Discussion}

The process of carcinogenesis and the early stage of tumor progression in ESCC are poorly understood. Frequent hypermethylation of $\mathrm{CpG}$ islands was previously observed in ESCC $(3,4,26)$. Epigenetic changes, including hypermethylation of the $\mathrm{CpG}$ island associated with a TRG, may result in the transcriptional silencing of a gene, with the subsequent loss of protein expression that may contribute to tumorigenesis (27). However, the majority of previous studies on the methylation of TRGs limited to analysis in surgically resected ESCC samples and did not study the status of TRG genes in early neoplastic lesions $(25,28)$. In the present study, IHC staining for 5 TRGs (FHIT, E-cadherin, MLH1, MSH2, and COX-2) was used to analyze methylation status, and it was identified that the expression of all 5 TRGs may be lost or reduced in cases of endoscopically resected early ESN. Additionally, ESN progression was indicated to be associated with the accumulation of methylation-modulated absence or reduced expression of the TRGs.

The expression of FHIT and E-cadherin appeared to be reduced or lost with the progression from LGIN to HGIN/CIS and then to invasive cancer. This finding is consistent with a previous study by the present authors (29) where the average number of the 5 TRG proteins with reduced or loss of expression in ESN showed a significant increase from LGIN to invasive cancer $(\mathrm{P}<0.05)$. Ishii et al $(30)$ reported that the accumulation of the DNA methylation of TRGs is associated with tumor progression in ESCC. Therefore, a continuous reduction or loss in the number of the TRG proteins expressed during ESCC progression may be an important mechanism of pathogenesis, not only for initial tumor development but also for the progression of ESCC.

$M L H 1$ and $M S H 2$ are post-replication mismatch repair genes (31). $M L H 1$ and $M S H 2$ promoter hypermethylation has been reported in 3-62\% $(15,32)$ and $29-32 \%(16,17)$ of ESCCs, respectively. In a previous study (28), the loss of MLH1 or MSH2 expression was detected in $28.7 \%$ of primary ESCCs 
that underwent radical esophagectomy and was significantly associated with increased malignancy as assessed by the increasing metastasis to lymph nodes, extent of invasion and decreasing level of tissue differentiation. There are only few studies in which abnormalities in the expression of $M L H 1$ and MSH2 genes were investigated in the early stage of ESCC, including from endoscopically resected specimens. In the present study, lost or reduced MLH1/MSH2 expression was detected in $0 \%(0 / 19)$ of LGIN cases, 7.1\% (5/70) of HGIN/CIS cases, and $11.5 \%(6 / 52)$ of invasive cancer cases, indicating that loss of $M L H 1 / M S H 2$ gene expression is involved in the carcinogenesis of some cases of ESCC.

$C O X-2$ overexpression has been associated with a strong inflammatory response and gastrointestinal carcinoma development (33). However, past studies have identified a low expression of the $C O X-2$ gene in gastric and colorectal cancer cases, associated with gene promoter hypermethylation $(34,35)$. Additionally, it has been reported that $C O X-2$ expression is regulated by promoter methylation in human esophageal cancer cell lines (36). However, in the present study, a low level of COX-2 expression was observed in $63.2 \%(12 / 19)$ of LGIN cases, $65.7 \%$ (46/70) of HGIN/CIS cases, and 59.6\% (31/52) of invasive cancer cases, therefore indicating no association between the loss of COX-2 expression and tumor progression.

Tobacco smoking and alcohol consumption are two major risk factors for ESCC, and there is increasing evidence for the ability of tobacco smoke-associated carcinogens and polymorphisms of carcinogen-metabolizing genes to modulate DNA methylation in certain types of tobacco-associated cancer, including lung and upper aerodigestive tract cancer $(37,38)$. Cigarette smoke was demonstrated to induce the promoter methylation of genes including FHIT and $C O X-2$ in ESCC $(14,23,39)$. E-cadherin promoter methylation was previously observed to be associated with increasing number of pack-years in HNC (40). There is also evidence to support the association of alcohol use with aberrant DNA methylation patterns in several types of cancer (20). ESCC has been suggested to be associated with promoter methylation of particular genes, including FHIT and MLH1 (39,41,42). In general, chronic inflammation induced by alcohol/tobacco use is considered an inducer of aberrant DNA methylation (43).

In the present study, expression of the $p 53$ gene was also analyzed. $p 53$ is a tumor-suppressor gene, and the p53 protein is crucial in a number of processes, including growth suppression, apoptosis and DNA repair (44). Tobacco and alcohol consumption may cause $p 53$ mutation, one of the most frequent events in esophageal carcinogenesis (45). Point mutations in the $p 53$ gene typically occur at an early stage of ESCC and correlate with tumor progression (46), therefore suggesting an important role of this genetic alteration in ESCC development. However, in the present study as well as a previous study, altered p53 expression was not significantly associated with the early stage of ESCC progression (47). From an epidemiological point of view, HNC and esophageal cancer are associated with the same carcinogens, including alcohol and tobacco $(45,48)$. Alterations in the $p 53$ gene and its expression have been reported in a variety of types of epithelial tumor and premalignant lesion, including HNC and esophageal cancer $(45,48)$. In the present study, the percentage of tumors with p53 overexpression was higher in ESNs with HNC compared with in ESN with other cancer.

In conclusion, methylation-modulated absent or reduced expression of 5 TRGs in the early stages of esophageal tumorigenesis was demonstrated. A loss or reduced protein expression of these TRGs was identified as associated with ESN progression. An improved understanding of the methylation modulation of TRGs will provide new insights into esophageal carcinogenesis, cancer treatment and reveal feasible targets for chemoprevention. Further investigations in this area are required to gain an improved insight into ESCC development and to support the development of novel therapeutic strategies.

\section{References}

1. Japan Esophageal Society: Comprehensive Registry of Esophageal Cancer in Japan, 3rd edition. 2002. Cited 1 May 2009. Available from URL: http://esophagus.jp

2. Muto M, Horimatsu T, Ezoe Y, Morita S and Miyamoto S: Improving visualization techniques by narrow band imaging and magnification endoscopy. J Gastroenterol Hepatol 24: 1333-1346, 2009.

3. Zhang XM and Guo MZ: The value of epigenetic markers in esophageal cancer. Front Med China 4: 378-384, 2010.

4. Ahrens TD, Werner M and Lassmann S: Epigenetics in esophageal cancers. Cell Tissue Res 356: 643-655, 2014.

5. Gao H, Wang LD, Zhou Q, Hong JY, Huang TY and Yang CS: p53 Tumor suppressor gene mutation in early esophageal precancerous lesions and carcinoma among high-risk populations in Henan. Cancer Res 54: 4342-4346, 1994.

6. Montesano R, Hollstein M and Hainaut P: Genetic alterations in esophageal cancer and their relevance to etiology and pathogenesis: A review. Int J Cancer 69: 225-235, 1996.

7. Abedi-Ardekani B, Dar NA, Mir MM, Zargar SA, Lone MM, Martel-Planche G, Villar S, Mounawar M, Saidi F, Malekzadeh R and Hainaut P: Epidermal growth factor receptor (EGFR) mutations and expression in squamous cell carcinoma of the esophagus in central Asia. BMC Cancer 12: 602, 2012.

8. Mori R, Ishiguro H, Kimura M, Mitsui A, Sasaki H, Tomoda K, Mori Y, Ogawa R, Katada T, Kawano O, et al: PIK3CA mutation status in Japanese esophageal squamous cell carcinoma. J Surg Res 145: 320-326, 2008.

9. Ushijima T: Detection and interpretation of altered methylation patterns in cancer cells. Nat Rev Cancer 5: 223-231, 2005.

10. Kalari S and Pfeifer GP: Identification of driver and passenger DNA methylation in cancer by epigenomic analysis. Adv Genet 70: 277-308, 2010.

11. Sato F and Meltzer SJ: CpG island hypermethylation in progression of esophageal and gastric cancer. Cancer 106: 483-493, 2006.

12. Mandelker DL, Yamashita K, Tokumaru Y, Mimori K, Howard DL, Tanaka Y, Carvalho AL, Jiang WW, Park HL, Kim MS, et al: PGP9.5 promoter methylation is an independent prognostic factor for esophageal squamous cell carcinoma. Cancer Res 65: 4963-4968, 2005.

13. Tzao C, Hsu HS, Sun GH, Lai HL, Wang YC, Tung HJ, Yu CP, Cheng YL and Lee SC: Promoter methylation of the hMLH1 gene and protein expression of human mutL homolog 1 and human mutS homolog 2 in resected esophageal squamous cell carcinoma. J Thorac Cardiovasc Surg 130: 1371, 2005.

14. Zhang GY, Ma CX, Liu QL, Le XP, Ding Y and Zhang QX: Detection of methylation of hMSH2 gene promoter region of esophageal cancer. Zhonghua Zhong Liu Za Zhi 27: 541-543, 2005 (In Chinese)

15. Lee EJ, Lee BB, Kim JW, Shim YM, Hoseok I, Han J, Cho EY, Park J and Kim DH: Aberrant methylation of fragile histidine triad gene is associated with poor prognosis in early stage esophageal squamous cell carcinoma. Eur J Cancer 42: 972-980, 2006.

16. Lee EJ, Lee BB, Han J, Cho EY, Shim YM, Park J and Kim DH: $\mathrm{CpG}$ island hypermethylation of E-cadherin (CDH1) and integrin alpha4 is associated with recurrence of early stage esophageal squamous cell carcinoma. Int J Cancer 123: 2073-2079, 2008.

17. Ling ZQ, Li P, Ge MH, Hu FJ, Fang XH, Dong ZM and Mao WM: Aberrant methylation of different DNA repair genes demonstrates distinct prognostic value for esophageal cancer. Dig Dis Sci 56: 2992-3004, 2011. 
18. Enzinger PC and Mayer RJ: Esophageal cancer. N Engl J Med 349: 2241-2252, 2003

19. Oze I, Matsuo K, Wakai K, Nagata C, Mizoue T, Tanaka K, Tsuji I, Sasazuki S, Inoue M and Tsugane S; Research Group for the Development and Evaluation of Cancer Prevention Strategies in Japan: Alcohol drinking and esophageal cancer risk: An evaluation based on a systematic review of epidemiologic evidence among the Japanese population. Jpn J Clin Oncol 41: 677-992, 2011

20. Varela-Rey M, Woodhoo A, Martinez-Chantar ML, Mato JM and Lu SC: Alcohol, DNA methylation, and cancer. Alcohol Res 35: 25-35, 2013.

21. Talukdar FR, Ghosh SK, Laskar RS and Mondal R: Epigenetic, genetic and environmental interactions in esophageal squamous cell carcinoma from northeast India. PLoS One 8: e60996, 2013

22. Portela A and Esteller M: Epigenetic modifications and human disease. Nat Biotechnol 28: 1057-1068, 2010.

23. Meng XY, Zhu ST, Zhou QZ, Li P, Wang YJ and Zhang ST: Promoter methylation regulates cigarette smoke-stimulated cyclooxygenase-2 expression in esophageal squamous cell carcinoma. J Dig Dis 13: 208-213, 2012.

24. Gabbert HE, Shimoda T, Hainaut P, Nakamura Y, Field JK and Inoue H: Squamous cell carcinoma of the oesophagus. In: Hamilton SR, Aaltonen LA, (eds). World Health Organization Classification of Tumours. Pathology and Genetics. Tumours of the Digestive System. Lyon: IARC Press, 8-19, 2000.

25. Kitamura A, Yashima K, Okamoto E, Andachi H, Hosoda A, Kishimoto Y, Shiota G, Ito H, Kaibara N and Kawasaki H: Reduced Fhit expression occurs in the early stage of esophageal tumorigenesis: No correlation with $\mathrm{p} 53$ expression and apoptosis. Oncology 61: 205-211, 2001.

26. Baba Y, Watanabe $M$ and Baba $\mathrm{H}$ : Review of the alterations in DNA methylation in esophageal squamous cell carcinoma. Surg Today 43: 1355-64, 2013.

27. Esteller M: CpG island hypermethylation and tumor suppressor genes: A booming present, a brighter future. Oncogene 21: 5427-5440, 2002 .

28. Uehara H, Miyamoto M, Kato K, Cho Y, Kurokawa T, Murakami S, Fukunaga A, Ebihara Y, Kaneko H, Hashimoto $\mathrm{H}$, et al: Deficiency of hMLH1 and hMSH2 expression is a poor prognostic factor in esophageal squamous cell carcinoma. J Surg Oncol 92: 109-115, 2005.

29. Hayashi A, Yashima K. Takeda Y, Sasaki S, Kawaguchi K, Harada K, Murawaki Y and Ito H: Fhit, E-cadherin, p53, and activation-induced cytidine deaminase expression in endoscopically resected early stage esophageal squamous neoplasia. J Gastroenterol Hepatol 27: 1752-1758, 2012.

30. Ishii T, Murakami J, Notohara K, Cullings HM, Sasamoto H, Kambara T, Shirakawa Y, Naomoto Y, Ouchida M, Shimizu K, et al: Oesophageal squamous cell carcinoma may develop within a background of accumulating DNA methylation in normal and dysplastic mucosa. Gut 56: 13-19, 2007.

31. Jiricny J: Replication errors: Challenging the genome. EMBO J 17: 6427-6436, 1998

32. Wang J, Sasco AJ, Fu C, Xue H, Guo G, Hua Z, Zhou Q, Jiang Q and $\mathrm{Xu}$ B: Aberrant DNA methylation of P16, MGMT, and hMLH1 genes in combination with MTHFR C677T genetic polymorphism in esophageal squamous cell carcinoma. Cancer Epidemiol Biomarkers Prev 17: 118-125, 2008.

33. Konturek PC, Kania J, Burnat G, Hahn EG and Konturek SJ: Prostaglandins as mediators of COX-2 derived carcinogenesis in gastrointestinal tract. J Physiol Pharmacol 56 (Suppl 5): S57-S73, 2005 .
34. Toyota M, Shen L, Ohe-Toyota M, Hamilton SR, Sinicrope FA and Issa JP: Aberrant methylation of the cyclooxygenase $2 \mathrm{CpG}$ island in colorectal tumors. Cancer Res 60: 4044-4048, 2000.

35. Huang L, Zhang KL, Li H, Chen XY, Kong QY, Sun Y, Gao X, Guan HW and Liu J: Infrequent COX-2 expression due to promoter hypermethylation in gastric cancers in Dalian, China. Hum Pathol 37: 1557-1567, 2006.

36. Meng XY, Zhu ST, Zong Y, Wang YJ, Li P and Zhang ST: Promoter hypermethylation of cyclooxy-genase-2 gene in esophageal squamous cell carcinoma. Dis Esophagus 24: 444-449, 2011.

37. Mani S, Szymańska K, Cuenin C, Zaridze D, Balassiano K, Lima SC, Matos E, Daudt A, Koifman S, Filho VW, et al: DNA methylation changes associated with risk factors in tumors of the upper aerodigestive tract. Epigenetics 7: 270-277, 2012.

38. Lin RK, Hsieh YS, Lin P, Hsu HS, Chen CY, Tang YA, Lee CF and Wang YC: The tobacco-specific carcinogen NNK induces DNA methyltransferase 1 accumulation and tumor suppressor gene hypermethylation in mice and lung cancer patients. J Clin Invest 120: 521-532, 2010.

39. Mori M, Mimori K, Shiraishi T, Alder H, Inoue H, Tanaka Y, Sugimachi K, Huebner K and Croce CM: Altered expression of Fhit in carcinoma precarcinomatous lesions of the esophagus. Cancer Res 60: 1177-1182, 2000.

40. Hasegawa M, Nelson HH, Peters E, Ringstrom E, Posner M and Kelsey KT: Patterns of gene promoter methylation in squamous cell cancer of the head and neck. Oncogene 21: 4231-4236, 2002.

41. Morita M, Oyama T, Nakata S, Ono K, Sugaya M, Uramoto H, Yoshimatsu T, Hanagiri T, Sugio K and Yasumoto K: Expression of fhit in esophageal epithelium and Carcinoma: Reference to drinking, smoking and multicentric carcinogenesis. Anticancer Res 26: 2243-2248, 2006.

42. van Engeland M, Weijenberg MP, Roemen GM, Brink M, de Bruïne AP, Goldbohm RA, van den Brandt PA, Baylin SB de Goeij AF and Herman JG: Effects of dietary folate and alcohol intake on promoter methylation in sporadic colorectal cancer: The Netherlands cohort study on diet and cancer. Cancer Res 63: 3133-3137, 2003.

43. Ushijima T and Okochi-Takada E: Aberrant methylations in cancer cells: Where do they come from? Cancer Sci 96: 206-211, 2005.

44. Hollestein M, Sidransky D, Vogelstein B and Harris CC: p53 mutations in human cancers. Science 253: 49-53, 1991.

45. Toh Y, Oki E, Ohgaki K, Sakamoto Y, Ito S, Egashira A, Saeki H, Kakeji Y, Morita M, Sakaguchi Y, et al: Alcohol drinking, cigarette smoking, and development of squamous cell carcinoma of the esophagus: Molecular mechanisms of carcinogenesis. Int J Clin Oncol 15: 135-144, 2010

46. Kaneko K, Katagiri A, Konishi K, Kurahashi T, Ito H, Kumekawa Y, Yamamoto T, Muramoto T, Kubota Y, Nozawa H, et al: Study of p53 gene alteration as a biomarker to evaluate the malignant risk of Lugol-unstained lesion with non-dysplasia in the oesophagus. Br J Cancer 96: 492-498, 2007.

47. Chino O, Kijima H, Shimada H, Nishi T, Tanaka H, Kise Y, Kenmochi T, Himeno S, Machimura T, Tanaka M, et al: Accumulation of p53 in esophageal squamous cell carcinoma. Int J Mol Med 8: 359-163, 2001.

48. Perez-Ordoñez B, Beauchemin M and Jordan RC: Molecular biology of squamous cell carcinoma of the head and neck. J Clin Pathol 59: 445-453, 2006. 